\title{
Palladium catalyzed hydroesterification of substituted alkenes under microwave conditions
}

\author{
Maretha du Plessis, Charlene Marais, and Barend C. B. Bezuidenhoudt* \\ Department of Chemistry, University of the Free State, P.O. Box 339, Bloemfontein, 9300, South Africa \\ Email: bezuidbc@ufs.ac.za, bcbbez@gmail.com
}

Received 03-18-2020

Accepted 06-22-2020

Published on line $07-02-2020$

\section{Abstract}

While several catalyst systems have been utilized in the hydroesterification or methoxycarbonylation of alkenes or equivalent substrates, these reactions are conventionally performed in autoclave reactor systems under high CO pressure (20 - 70 bar) and thermal heating $\left(70-110{ }^{\circ} \mathrm{C}\right)$. In this paper, the first methoxycarbonylation reactions performed in a microwave reactor fitted with a gas-addition accessory system are reported on and compared to the same reactions performed under conventional heating in an autoclave reactor. Thus 1 -octene, styrene, allylbenzene, $o$ - and $p$-methoxyallylbenzene and $\beta$-methylstyrene were subjected to methoxycarbonylation over a palladium acetate-aluminum triflate catalyst system at 12 bar and $95{ }^{\circ} \mathrm{C}$. Results obtained indicated the methoxycarbonylation of these alkenes to be much faster under microwave conditions when compared to conventional heating and improvements in conversion ranged between 3 and $5 \%$ for the more reactive substrates (1-octene and styrene) and $6-20 \%$ for the allylbenzenes and $\beta$-methylstyrene.

$$
\begin{aligned}
& \mathrm{Pd}(\mathrm{OAc})_{2}(5 \mathrm{~mol} \%) \\
& \begin{array}{ll}
\mathrm{R}^{1} \mathrm{R}^{2} & \begin{array}{l}
\mathrm{Al}(\mathrm{OTf})_{3}(10 \mathrm{~mol} \%) \\
\mathrm{PPh}_{3}(20 \mathrm{~mol} \%)
\end{array} \\
\cline { 2 - 2 } \mathrm{R}^{1}=\left(\mathrm{CH}_{2}\right)_{5} \mathrm{CH}_{3}, \mathrm{Ph} \text { or } \mathrm{Bn} & \mathrm{MeOH}, \mathrm{CO} \\
\mathrm{R}^{2}=\mathrm{H} \text {, or } \mathrm{CH}_{3} \mathrm{C} C
\end{array} \\
& \mathrm{R}^{1} \mathrm{R}^{2} \\
& \mathrm{R}^{1}=\left(\mathrm{CH}_{2}\right)_{5} \mathrm{CH}_{3} \text {, Ph or Bn } \\
& \mathrm{R}^{2}=\text { COOMe, } \mathrm{R}^{3}=\mathrm{H}(I) \\
& \mathrm{R}^{2}=\mathrm{H}, \mathrm{R}^{3}=\stackrel{\text { or }}{\text { coome }}(b) \\
& \text { or } \\
& \mathrm{R}^{2}=\mathrm{CH}_{3}, \mathrm{R}^{3}=\text { COOMe }(b n)
\end{aligned}
$$

Keywords: Alkoxycarbonylation, hydroesterification, palladium catalyst, aluminum triflate, alkenes, microwave heating. 


\section{Introduction}

While the palladium catalyzed alkoxycarbonylation or hydroesterification of alkenes or equivalent substrates has been well documented for a plethora of catalyst systems, ${ }^{1-16}$ these reactions are usually performed in autoclave reactors at $\mathrm{CO}$ pressures ranging from ca. $1-70$ bar and temperatures of ca. $70-110^{\circ} \mathrm{C}$. During an investigation into the electronic factors governing the regioselectivity (ratio of linear to branched to benzylic products) of the addition of the ester entity to aromatic substituted alkenes over a palladium catalyst system, ${ }^{17}$ it was found that the reactivity of the substituted allylbenzene (1) and $\beta$-methylstyrene (2) reactants were rather low [Turn-over frequencies (TOF) of $7-30 \mathrm{~h}^{-1}$ ], so prolonged reactions times (up to $3 \mathrm{~h}$ ) were required to obtain decent yields (50-90\%).

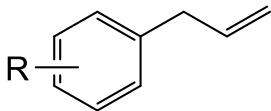

1

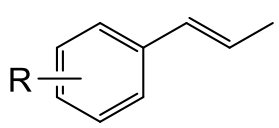

2

Since it is generally known that microwave heating has the ability to accelerate some chemical reactions ${ }^{18}$ and the hydroesterification reaction was found to be rather slow, it was decided to evaluate the effect of microwave heating on the rate of the alkoxycarbonylation of substituted alkenes over a palladium acetate catalyst system.

\section{Results and Discussion}

In order to be in a position to compare the results of the microwave mediated reactions with the results obtained under conditions of ordinary heating at $95{ }^{\circ} \mathrm{C}$ and the optimum pressure of $35 \mathrm{bar}$, as reported earlier, ${ }^{5}$ the same $\mathrm{Pd}(\mathrm{II}) / \mathrm{Al}(\mathrm{OTf})_{3} / \mathrm{PPh}_{3}$ catalyst system, developed by Williams et al., ${ }^{19,20}$ was utilized during the current investigation. Furthermore, since the glass vessels of the microwave reactor are rated at 12 bar only, the reactions in an autoclave reactor were also performed at 12 bar $\mathrm{CO}$ pressure $\left(95^{\circ} \mathrm{C}\right)$, while the investigation was started with the very reactive general hydroesterification substrates, 1-octene (3) and styrene (4) (Scheme 1) for possible comparison with literature results.

$$
\begin{aligned}
& \mathrm{Pd}(\mathrm{OAc})_{2}(5 \mathrm{~mol} \%) \\
& \mathrm{Al}(\mathrm{OTf})_{3}(10 \mathrm{~mol} \%) \\
& \mathrm{R}^{1} \mathrm{R}^{2}
\end{aligned}
$$

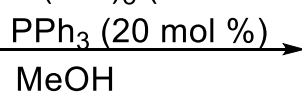

$$
\begin{aligned}
& 3 \mathrm{R}^{1}=\left(\mathrm{CH}_{2}\right)_{5} \mathrm{CH}_{3}, \mathrm{R}^{2}=\mathrm{H} \quad \mathrm{CO} \\
& 95^{\circ} \mathrm{C} \\
& \mathrm{R}_{\mathrm{R}^{3}}^{1} \mathrm{R}^{2} \\
& 4 \mathrm{R}^{1}=\mathrm{Ph}, \mathrm{R}^{2}=\mathrm{H} \\
& 5 \mathrm{R}^{1}=\left(\mathrm{CH}_{2}\right)_{5} \mathrm{CH}_{3}, \mathrm{R}^{2}=\mathrm{COOMe}, \mathrm{R}^{3}=\mathrm{H}(I) \\
& 6 \mathrm{R}^{1}=\left(\mathrm{CH}_{2}\right)_{5} \mathrm{CH}_{3}, \mathrm{R}^{2}=\mathrm{H}, \mathrm{R}^{3}=\text { COOMe }(b) \\
& 7 \mathrm{R}^{1}=\mathrm{Ph}, \mathrm{R}^{2}=\mathrm{COOMe}, \mathrm{R}^{3}=\mathrm{H}(I) \\
& 8 \mathrm{R}^{1}=\mathrm{Ph}, \mathrm{R}^{2}=\mathrm{H}, \mathrm{R}^{3}=\text { COOMe }(b)
\end{aligned}
$$

Scheme 1. Methoxycarbonylation of 1-octene (3) and styrene (4). I and $b$ refers to the 'linear' and 'branched' isomers, respectively. 
As indicated in Table 1, both substrates, 1-octene (3) and styrene (4) showed a substantial increase in conversion of ca. $20 \%$ and $30 \%$, respectively, even after only 10 minutes of reaction time for the microwave reaction when compared to the autoclave reaction at the same pressure (12 bar). Furthermore, virtually complete conversion of the starting material (99\%) was observed after 30 minutes under microwave irradiation, while the reactions under conventional heating conditions at the same pressure (12 bar) were not fully completed (Table 1, entries 2 vs. 3 and 5 vs. 6). Even when compared to the reactions at 35 bar, the microwave equivalents showed a slight improvement over those performed with conventional heating (Table 1 , entries 1 vs. 3 and 4 vs. 6). The fact that the final yields for the microwave reactions are somewhat lower than those of the reactions in the autoclave reactor under the same conditions $(82 \%$ vs. $91 \%$ and $72 \%$ vs. $92 \%$, respectively) is probably explicable in terms of unwanted side-reactions. These could include reactions like polymerization in the case of styrene (4), and/or subsequent hydrolysis of the ester products, 5, 6, $\mathbf{7}$ and $\mathbf{8}$, by water present in the methanol, due to the extended exposure to the microwave heating conditions, since the MW reactions were already $99 \%$ and $89 \%$ completed after only 10 minutes (Table 1, entries 3 and 6).

Table 1. Methoxycarbonylation of 1-octene (3) and styrene (4)

\begin{tabular}{|c|c|c|c|c|c|c|c|c|c|}
\hline \multirow[t]{2}{*}{ Entry } & \multirow[t]{2}{*}{ Substrate } & \multirow{2}{*}{$\begin{array}{c}\text { CO Pressure } \\
\text { (bar) }\end{array}$} & \multirow{2}{*}{$\begin{array}{c}\text { Temp. } \\
\left({ }^{\circ} \mathrm{C}\right)\end{array}$} & \multicolumn{2}{|c|}{ Conversion $^{c}(\%)$} & \multirow[t]{2}{*}{ Products } & \multicolumn{2}{|c|}{ Yield $^{d}(\%)$} & \multirow{2}{*}{$\begin{array}{c}\text { Ratio } \\
(I: b)\end{array}$} \\
\hline & & & & $10 \mathrm{~min}$ & $30 \mathrm{~min}$ & & $10 \mathrm{~min}$ & $30 \mathrm{~min}$ & \\
\hline 1 & 3 & 35 & $95^{a}$ & 85 & 95 & $5 \& 6$ & 76 & 92 & $4: 1$ \\
\hline 2 & 3 & 12 & $95^{a}$ & 80 & 94 & $5 \& 6$ & 70 & 91 & $4: 1$ \\
\hline 3 & 3 & 12 & $\begin{array}{c}95 \\
(\mathrm{MW})^{\mathrm{b}}\end{array}$ & 99 & 99 & $5 \& 6$ & 82 & 82 & $4: 1$ \\
\hline 4 & 4 & 35 & $95^{a}$ & 77 & 96 & $7 \& 8$ & 64 & 93 & $4: 1$ \\
\hline 5 & 4 & 12 & $95^{a}$ & 58 & 96 & $7 \& 8$ & 53 & 92 & $4: 1$ \\
\hline 6 & 4 & 12 & $\begin{array}{c}95 \\
(\mathrm{MW})^{\mathrm{b}}\end{array}$ & 89 & 99 & $7 \& 8$ & 71 & 72 & $4: 1$ \\
\hline
\end{tabular}

${ }^{a}$ Conventional heating in an autoclave reactor. ${ }^{b}$ Microwave irradiation (150 W). ${ }^{c}$ Determined by GC analysis with xylene as internal standard. ${ }^{\mathrm{d}}$ Combined yield determined by GC analysis (with xylene as internal standard).

When subjecting the aryl substituted alkenes, allylbenzene (9), 4-allylanisole (10), 2-allylanisole (11) and trans- $\beta$-methylstyrene (12) to the methoxycarbonylation reaction, it should be kept in mind that these substrates are prone to palladium catalyzed isomerization of the double bond between the internal and terminal positions, so the same products, i.e. 'linear', 'branched' and 'benzylic' isomers, could be formed from both the similarly substituted allylbenzene and $\beta$-methylstyrene derivatives (Scheme 2 ). 


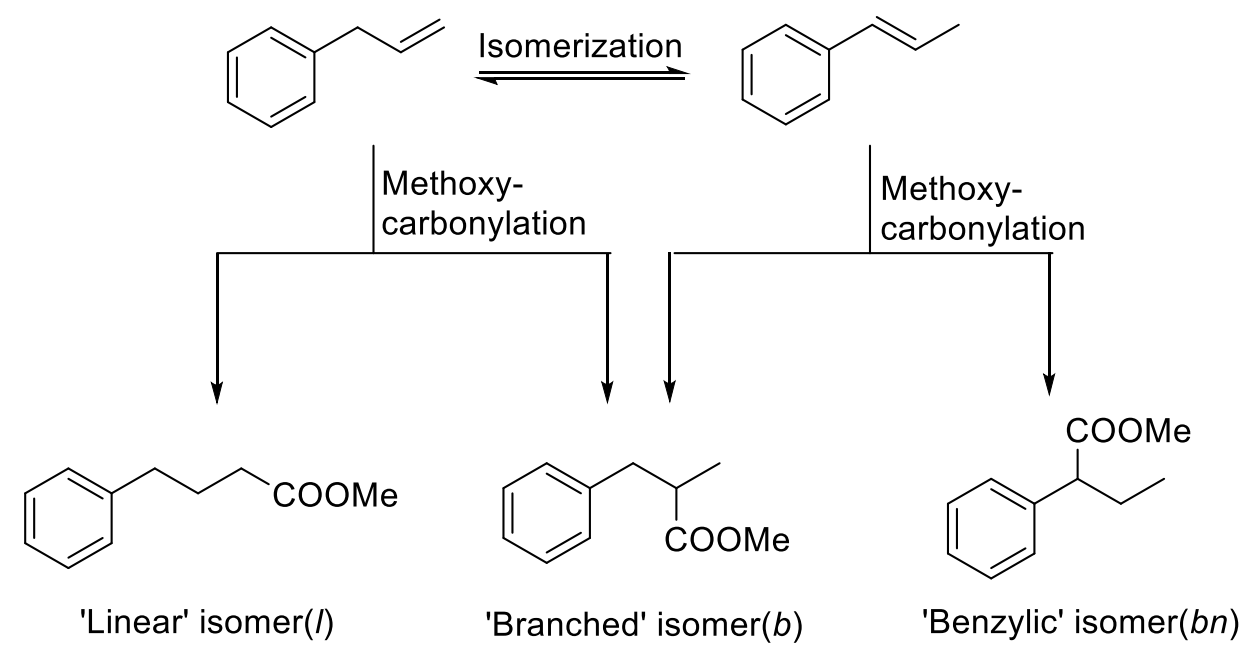

Scheme 2. Methoxycarbonylation and isomerization of allylbenzene (9) and $\beta$-methylstyrene (12).

When the 1- and 2-propenylaryl substrates (Scheme 3) were subjected to the methoxycarbonylation reaction under microwave heating $\left(150 \mathrm{~W}\right.$ ) over the standard catalyst system, $\mathrm{Pd}(\mathrm{OAc})_{2} / \mathrm{Al}(\mathrm{OTf})_{3} / \mathrm{PPh}_{3}$, it was found that all the substrates reacted much slower than 1-octene (3) and styrene (4), so reaction times of up to three hours were required to reach completion in both the $\mathrm{MW}$ and autoclave reactions at 35 bar. $p$ Methoxyallylbenzene (10) proved to be even less reactive and gave conversions of only $40-60 \%$ after the 3 hours, with the better conversion (60\%) observed for the autoclave reaction at 35 bar. When comparing the reactions at 12 bar for this substrate (10), however, the MW method gave a better conversion (28\% vs. 20\%) even after only 1 hour (Table 2 , entry 5 vs. 6).
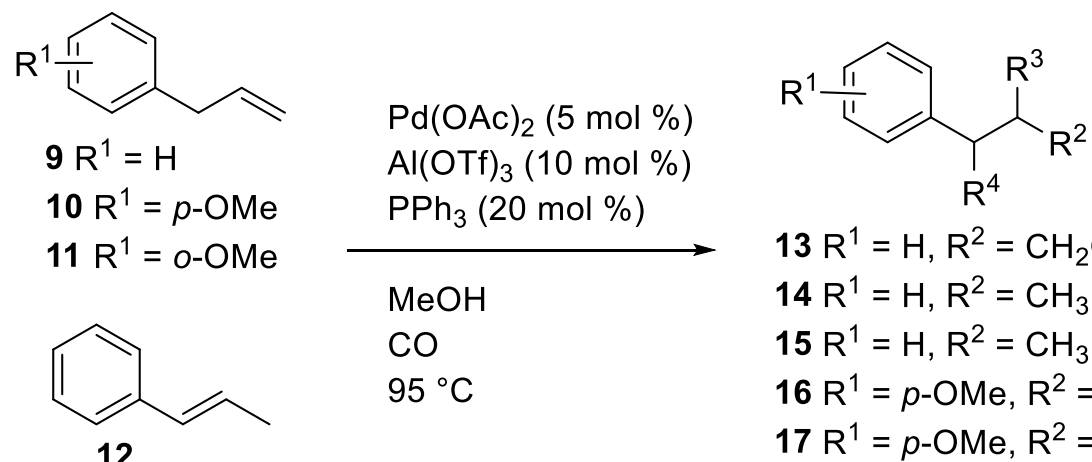

$$
\begin{aligned}
& 13 \mathrm{R}^{1}=\mathrm{H}, \mathrm{R}^{2}=\mathrm{CH}_{2} \text { COOMe, } \mathrm{R}^{3}=\mathrm{R}^{4}=\mathrm{H}(l) \\
& 14 \mathrm{R}^{1}=\mathrm{H}, \mathrm{R}^{2}=\mathrm{CH}_{3}, \mathrm{R}^{3}=\mathrm{COOMe}, \mathrm{R}^{4}=\mathrm{H}(b) \\
& 15 \mathrm{R}^{1}=\mathrm{H}, \mathrm{R}^{2}=\mathrm{CH}_{3}, \mathrm{R}^{3}=\mathrm{H}, \mathrm{R}^{4}=\mathrm{COOMe}(b n) \\
& 16 \mathrm{R}^{1}=p-O M e, \mathrm{R}^{2}=\mathrm{CH}_{2} \text { COOMe, } \mathrm{R}^{3}=\mathrm{R}^{4}=\mathrm{H}(l) \\
& 17 \mathrm{R}^{1}=p-O M e, \mathrm{R}^{2}=\mathrm{CH}_{3}, \mathrm{R}^{3}=\mathrm{COOMe}, \mathrm{R}^{4}=\mathrm{H}(b) \\
& 18 \mathrm{R}^{1}=p-O M e, \mathrm{R}^{2}=\mathrm{CH}_{3}, \mathrm{R}^{3}=\mathrm{H}, \mathrm{R}^{4}=\mathrm{COOMe}(b n) \\
& 19 \mathrm{R}^{1}=\text { o-OMe, } \mathrm{R}^{2}=\mathrm{CH}_{2} \text { COOMe, } \mathrm{R}^{3}=\mathrm{R}^{4}=\mathrm{H}(l) \\
& 20 \mathrm{R}^{1}=\text { o-OMe, } \mathrm{R}^{2}=\mathrm{CH}_{3}, \mathrm{R}^{3}=\mathrm{COOMe}, \mathrm{R}^{4}=\mathrm{H}(b) \\
& 21 \mathrm{R}^{1}=\text { o-OMe, } \mathrm{R}^{2}=\mathrm{CH}_{3}, \mathrm{R}^{3}=\mathrm{H}, \mathrm{R}^{4}=\mathrm{COOMe}(b n)
\end{aligned}
$$

Scheme 3. Methoxycarbonylation of allylbenzenes $(\mathbf{9}-\mathbf{1 1})$ and $\beta$-methylstyrene (12).

A substantial improvement in conversion ( $70 \%$ vs. $86 \%$ and $29 \%$ vs. $65 \%$, respectively) was also obtained for the 0 -methoxy substituted allylbenzene analogue, 11 and $\beta$-methylstyrene (12), during conditions of MW irradiation over conventional heating after 1 hour at the same pressure (Table 2, entries 8 vs. 9 and 11 vs 12). After 3 hours at 12 bar (at nearly full conversion), improved conversion rates were obtained for all substrates 
(9 - 12), with the more profound improvements (ca. 20\%) for the less reactive substrates, $p$-methoxyallylbenzene (10) and $\beta$-methylstyrene (12), (Table 2, entries 2 vs 3, 5 vs. 6,8 vs 9 and 11 vs 12 respectively).

Table 2. Methoxycarbonylation of allylbenzenes (9 - 11) and $\beta$-methylstyrene (12)

\begin{tabular}{|c|c|c|c|c|c|c|c|c|}
\hline \multirow[t]{2}{*}{ Entry } & \multirow[t]{2}{*}{ Substrate } & \multirow{2}{*}{$\begin{array}{l}\text { CO Pressure } \\
\text { (bar) }\end{array}$} & \multirow{2}{*}{$\begin{array}{l}\text { Temp. } \\
\left({ }^{\circ} \mathrm{C}\right)\end{array}$} & \multicolumn{2}{|c|}{ Conversion $^{c}(\%)$} & \multirow[t]{2}{*}{ Products } & \multirow{2}{*}{$\begin{array}{c}\text { Yield }^{d} \\
(\%)\end{array}$} & \multirow{2}{*}{$\begin{array}{l}\text { Ratio } \\
\text { (l:b:bn) }\end{array}$} \\
\hline & & & & $1 \mathrm{~h}$ & $3 \mathrm{~h}$ & & & \\
\hline 1 & 9 & 35 & $95^{a}$ & 72 & 97 & $13,14,15$ & 80 & $7: 2: 1$ \\
\hline 2 & 9 & 12 & $95^{\mathrm{a}}$ & 57 & 87 & $13,14,15$ & 80 & $12: 2: 1$ \\
\hline 3 & 9 & 12 & $95(\mathrm{MW})^{\mathrm{b}}$ & 57 & 90 & $13,14,15$ & 81 & $9: 2: 1$ \\
\hline 4 & 10 & 35 & $95^{a}$ & 38 & 60 & $16,17,18$ & 51 & $5: 2: 1$ \\
\hline 5 & 10 & 12 & $95^{a}$ & 20 & 21 & $16,17,18$ & 20 & $15: 2: 1$ \\
\hline 6 & 10 & 12 & $95(\mathrm{MW})^{\mathrm{b}}$ & 28 & 40 & $16,17,18$ & 37 & $6: 1: 1$ \\
\hline 7 & 11 & 35 & $95^{a}$ & 88 & 96 & $19,20,21$ & 75 & $26: 5: 1$ \\
\hline 8 & 11 & 12 & $95^{a}$ & 70 & 90 & $19,20,21$ & 73 & $28: 6: 1$ \\
\hline 9 & 11 & 12 & $95(\mathrm{MW})^{\mathrm{b}}$ & 86 & 96 & $19,20,21$ & 89 & $27: 5: 1$ \\
\hline 10 & 12 & 35 & $95^{a}$ & 47 & 95 & $13,14,15$ & 76 & $5: 2: 1$ \\
\hline 11 & 12 & 12 & $95^{\mathrm{a}}$ & 29 & 74 & $13,14,15$ & 66 & $10: 2: 1$ \\
\hline 12 & 12 & 12 & $95(\mathrm{MW})^{\mathrm{b}}$ & 65 & 94 & $13,14,15$ & 82 & $8: 2: 1$ \\
\hline
\end{tabular}

${ }^{\mathrm{a}}$ Conventional heating in an autoclave reactor. ${ }^{\mathrm{b}}$ Microwave irradiation ( $\left.150 \mathrm{~W}\right)$. ${ }^{\mathrm{C}}$ Determined by GC analysis with xylene as internal standard. ${ }^{\mathrm{d}}$ Combined yield determined by GC analysis (with xylene as internal standard) after 3 hours.

A comparison of the final product yields revealed that, apart from allylbenzene (9), MW irradiation resulted in substantial increases (ca. 16\%) for all substrates when compared to the conventional thermal reactions at the same pressure (12 bar) (table 2, entries 5 vs 6,8 vs 9 and 11 vs 12 , respectively).

With regard to the regioselectivity of the reactions, a general decrease in selectivity was evident in the microwave reactions when compared to the conventional heating experiments at the same $\mathrm{CO}$ pressure (Table 2, entry 2 vs. 3, 5 vs. 6,8 vs. 9 and 11 vs. 12). Though still the major products, the lower selectivity towards the linear products is probably explicable in terms of a more facile isomerization to the corresponding internal olefins for the allylbenzene substrates under the microwave conditions, while isomerization of the $\beta$ methylstyrene to the sterically less hindered allylbenzene isomer may be slower than the secondary terminal to internal isomerization.

\section{Conclusions}

Finally, for 1-octene (3) and styrene (4), a definite reaction rate increasing effect was observed with the conversion after 10 minutes under microwave conditions at 12 bar being almost the same or better than what was obtained after 30 minutes at 35 bar with conventional heating in an autoclave reactor. Although no dramatic reaction rate enhancing effect was observed for the other substrates, both the substituted allylbenzene analogues (10 and $\mathbf{1 1}$ ) and $\beta$-methylstyrene (12) showed improvements in conversion and yield under microwave conditions when compared to conventional heating at the same pressure. The fact that only 
a very slight improvement in conversion and yield could be detected for allylbenzene (9) is currently inexplicable.

\section{Experimental Section}

General. All the chemicals used were purchased from Sigma-Aldrich and used as received. Reactions were performed in a 4590 Parr autoclave reactor $(25 \mathrm{ml}$ vessel) with mechanical stirring (500 rpm) and a CEM Discover SP Microwave Reactor, equipped with a gas addition accessory and an internal IR temperature sensor, in a sealed tube (10 ml tube) and stirred by a magnetic stirrer bar. ${ }^{1} \mathrm{H}$ and ${ }^{13} \mathrm{C}$ NMR as well as $2 \mathrm{D}$ experiments were done on a $600 \mathrm{MHz}$ Bruker AM FT spectrometer at $20^{\circ} \mathrm{C}$ at $600 \mathrm{MHz}$ for ${ }^{1} \mathrm{H}$ NMR and 151 $\mathrm{MHz}$ for ${ }^{13} \mathrm{C}$ in $\mathrm{CDCl}_{3}$ as solvent. ${ }^{1} \mathrm{H}$ NMR chemical shifts are given relative to the TMS signal at $0 \mathrm{ppm}$ and ${ }^{13} \mathrm{C}$ NMR shifts are given relative to the $\mathrm{CDCl}_{3}$ peak at $77.16 \mathrm{ppm}$, coupling constants are measured in $\mathrm{Hz}$ and chemical shifts in ppm. Mass spectrometry was performed on a Shimadzu GCMS QP-2010 instrument using electron-impact (EI) ionization. Conversions, yields and product ratios were determined on a Shimadzu 2010 GC instrument with xylene as the internal standard. Methyl nonanoate (5) and methyl 2-methyloctanoate (6) were purified by passing the reaction mixture through a pad of silica gel, while all the other products were purified using flash column chromatography with silica gel 60 as stationary phase and obtained as mixtures of linear, branched and, in the case of the allylbenzenes, benzylic isomers.

Autoclave procedure. $\mathrm{Pd}(\mathrm{OAc})_{2}(5 \mathrm{~mol} \%), \mathrm{Al}(\mathrm{OTf})_{3}(10 \mathrm{~mol} \%), \mathrm{PPh}_{3}(20 \mathrm{~mol} \%), \mathrm{xylene}(0.5 \mathrm{~g})$ and the alkene $(4$ $\mathrm{mmol})$ were added together in the Parr autoclave reactor and dissolved in $\mathrm{MeOH}(8 \mathrm{ml})$. The mechanical stirrer was activated, the reactor purged with $\mathrm{CO}$ (purified by passing the gas through a $\mathrm{CrO}_{3} \mathrm{Column}$ and allowed to heat up to $95^{\circ} \mathrm{C}$, where after it was pressurized to 12 bar with CO.

Microwave procedure. $\mathrm{Pd}(\mathrm{OAc})_{2}$ (5 mol\%), Al(OTf) 3 (10 mol\%), $\mathrm{PPh}_{3}(20 \mathrm{~mol} \%)$, xylene $(0.25 \mathrm{~g})$ and the alkene $(2 \mathrm{mmol})$ were added together in a microwave vial $(10 \mathrm{ml})$ and dissolved in $\mathrm{MeOH}(4 \mathrm{ml})$. The magnetic stirrer was started and the reactor purged with $\mathrm{CO}$ (purified by passing the gas through a $\mathrm{CrO}_{3}$ column) and the $\mathrm{CO}$ pressure set to 12 bar, where after irradiation at $150 \mathrm{~W}$ was initiated and the temperature kept at $95{ }^{\circ} \mathrm{C}$ by utilizing the fixed power method.

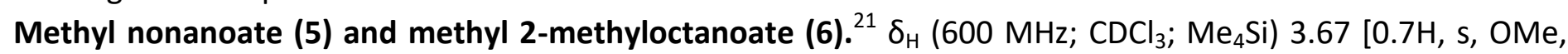
(6)], $3.66[3 \mathrm{H}, \mathrm{s}, \mathrm{OMe},(5)], 2.44-2.41[0.2 \mathrm{H}, \mathrm{m}, \mathrm{H}-2,(6)], 2.29[2 \mathrm{H}, \mathrm{t}, \mathrm{J} 7.57 \mathrm{~Hz}, \mathrm{H}-2,(5)], 1.64-1.59[2 \mathrm{H}, \mathrm{m}, \mathrm{H}-3$, (5)], 1.32-1.22 [10H, m, H-4, H-5, H-6, H-7, H-8 (5)], 1.32-1.22 [2.2H, m, H-3, H-4, H-5, H-6, H-7, (6)], 1.13 [0.7H, d, J $\left.7.02 \mathrm{~Hz}, 2-\mathrm{CH}_{3},(6)\right], 0.87[0.7 \mathrm{H}, \mathrm{t}, J 7.02 \mathrm{~Hz}, \mathrm{H}-8,(6)], 0.87[3 \mathrm{H}, \mathrm{t}, J 7.02 \mathrm{~Hz}, \mathrm{H}-9,(5)] ; \delta_{\mathrm{C}}\left(151 \mathrm{MHz} ; \mathrm{CDCl}_{3}\right)$ 177.5 [C-1 (6)], 174.4 [C-1 (5)], 51.5 [OMe (5)], 51.3 [OMe (6)], 39.6 [C-2 (6)], 34.19 [C-2 (5)], 33.9 (6), 32.0 (5), 31.8, (6), $29.3(5), 29.3(6), 29.3(5), 29.2(5), 27.3(6), 25.1[\mathrm{C}-3,(5)], 22.7(5), 22.7(6), 17.2$ [2-CH,$(6)], 14.2$ $[\mathrm{C}-9,(5)], 14.1[\mathrm{C}-8,(6)] ; \mathrm{MS}(\mathrm{EI}) \mathrm{m} / z 172\left[\mathrm{M}^{+}, 2 \%,(5)\right], 172\left[\mathrm{M}^{+}, 1 \%,(6)\right]$.

Methyl 3-phenylpropanoate ${ }^{22}$ (7) and methyl 2-phenylpropanoate ${ }^{23}$ (8). $R_{f}=0.595$ (petroleum ether:ether 7:3); $\delta_{\mathrm{H}}\left(600 \mathrm{MHz} ; \mathrm{CDCl}_{3} ; \mathrm{Me}_{4} \mathrm{Si}\right)$ 7.34-7.26 [2.7H, m, H-Ar, (7) and (8)], 7.22-7.19 [2.9H, m, CH aromatic, (7) and (8)], $3.72[0.2 \mathrm{H}, \mathrm{q}, J 7.17 \mathrm{~Hz}, \mathrm{H}-2,(8)], 3.67[3 \mathrm{H}, \mathrm{s}, \mathrm{OMe},(7)], 3.65[0.5 \mathrm{H}, \mathrm{s}, \mathrm{OMe},(8)], 2.95[2 \mathrm{H}, \mathrm{t}, J 7.89 \mathrm{~Hz}$, $\mathrm{H}-3,(7)] 2.63[2 \mathrm{H}, \mathrm{t}, J 7.89 \mathrm{~Hz}, \mathrm{H}-2,(7)], 1.50[0.5 \mathrm{H}, \mathrm{d}, J 7.17 \mathrm{~Hz}, \mathrm{H}-3,(8)] ; \delta_{\mathrm{C}}\left(151 \mathrm{MHz} ; \mathrm{CDCl}_{3}\right) 175.1[\mathrm{C}-1,(8)]$, $173.5[\mathrm{C}-1,(7)], 140.7\left[\mathrm{C}-1^{\prime},(8)\right], 140.6$ [C-1', (7)], 128.8 [C-4', (8)], 128.6 [CH aromatic, (7)], 128.4 [CH aromatic, (7)], 127.6 [CH aromatic, (8)], 127.3 [CH aromatic, (8)], 126.4 [C-4' (7)], 52.2 [-OMe, (8)], 51.7 [-OMe (7)], 45.6 [C-2, (8)], 35.8 [C-2, (7)], 31.1 [C-3 (7)], 18.7 [C-3, (8)]; MS (EI) m/z 164 [M+, 35\%, (7)], 164 [M+, 23\%, (8)]. 
Methyl 4-phenylbutanoate ${ }^{12}$ (13), methyl 2-methyl-3-phenylpropanoate ${ }^{24}$ (14) and methyl 2phenylbutanoate ${ }^{25}(\mathbf{1 5}) . \quad \mathrm{R}_{\mathrm{f}}=0.784$ (hexane:toluene:acetone 4:4:2); $\delta_{\mathrm{H}}\left(600 \mathrm{MHz} ; \mathrm{CDCl}_{3} ; \mathrm{Me}_{4} \mathrm{Si}_{\text {) }} 7.33-7.24\right.$ $[4.8 \mathrm{H}, \mathrm{m}, \mathrm{CH}$ aromatic, (13), (14) and (15)], 7.22-7.15 [4.5H, m, CH aromatic, (13), (14) and (15)], 3.66 [3H, s, OMe (13)], $3.65[1 \mathrm{H}, \mathrm{s}, \mathrm{OMe}(15)], 3.64[1.7 \mathrm{H}, \mathrm{s}, \mathrm{OMe}(14)], 3.46[0.3 \mathrm{H}, \mathrm{t}, J 7.71 \mathrm{~Hz}, \mathrm{H}-2,(15)], 3.03[0.6 \mathrm{H}, \mathrm{dd}, J$ 6.83 and $13.37 \mathrm{~Hz}, \mathrm{H}-3 \mathrm{a} / \mathrm{b},(14)], 2.77-2.71[0.6 \mathrm{H}, \mathrm{m}, \mathrm{H}-2,(14)], 2.66[0.6 \mathrm{H}, \mathrm{dd}, J 7.83$ and $13.37 \mathrm{~Hz}, \mathrm{H}-3 \mathrm{a} / \mathrm{b}$, (14)], $2.65[2 \mathrm{H}, \mathrm{t}, J 7.56 \mathrm{~Hz}, \mathrm{H}-4,(13)], 2.33[2 \mathrm{H}, \mathrm{t}, J 7.56 \mathrm{~Hz}, \mathrm{H}-2,(13)], 2.14-2.06[0.3 \mathrm{H}, \mathrm{m}, \mathrm{H}-3 \mathrm{a} / \mathrm{b},(15)], 1.96$ [2H, p, J $7.56 \mathrm{~Hz}, \mathrm{H}-3,(13)], 1.83-1.76[0.3 \mathrm{H}, \mathrm{m}, \mathrm{H3a} / \mathrm{b},(15)], 1.15\left[1.7 \mathrm{H}, \mathrm{d}, J 6.92 \mathrm{~Hz}, 2-\mathrm{CH}_{3},(14)\right], 0.94[1 \mathrm{H}, \mathrm{t}, J$ $7.37 \mathrm{~Hz}, \mathrm{H}-4,(15)] ; \delta_{\mathrm{C}}\left(151 \mathrm{MHz} ; \mathrm{CDCl}_{3}\right) 176.7$ [C-1, (14)], 174.7 [C-1, (15)], 174.1 [C-1, (13)], 141.5 [C-1', (13)], 139.5 [C-1', (14)], 139.2 [C-1', (15)], 129.1, 128.7, 128.6, 128.51, 128.48, 128.1, 127.3, 126.4, 126.1, 53.5 [C-2, (15)], 52.0 [OMe, (15], 51.72 [OMe, (14)], 51.65 [OMe (13)], 41.6 [C-2, (14)], 39.8 [C-3 (14)], 35.2 [C-4, (13)], $33.5[\mathrm{C}-2,(\mathbf{1 3})], 26.9$ [C-3 (15)], $26.6[\mathrm{C}-3,(\mathbf{1 3})], 16.9$ [2- $\left.\mathrm{CH}_{3}(\mathbf{1 4})\right], 12.3[\mathrm{C}-4,(\mathbf{1 5})] ; \mathrm{MS}(\mathrm{EI}) \mathrm{m} / z 178\left[\mathrm{M}^{+}, 37 \%\right.$, (13)], $178\left[\mathrm{M}^{+}, 18 \%,(14)\right], 178\left[\mathrm{M}^{+}, 18 \%,(15)\right]$.

Methyl 4-(4'-methoxyphenyl)butanoate ${ }^{12}$ (16), methyl 3-(4'-methoxyphenyl)-2-methylpropanoate ${ }^{24}(17)$ and methyl 2-(4'-methoxyphenyl)butanoate ${ }^{26}$ (18). $\mathrm{R}_{\mathrm{f}} 0.639$ (petroleum ether:ether 7:3); $\delta_{\mathrm{H}}\left(600 \mathrm{MHz} \mathrm{CDCl}_{3}\right.$; $\left.\mathrm{Me}_{4} \mathrm{Si}\right) 7.25\left[0.2 \mathrm{H}, \mathrm{d}, J 8.7 \mathrm{~Hz}, \mathrm{H}-3^{\prime}\right.$ and H-5', (18)], 7.11 [2H, d, J $8.66 \mathrm{~Hz}, \mathrm{H}-3^{\prime}$ and H-5', (16)], 7.10 [0.4H, d, J $8.64 \mathrm{~Hz}, \mathrm{H}-3^{\prime}$ and $\mathrm{H}^{-5}$ ', (17)], $6.88\left[0.2 \mathrm{H}, \mathrm{d}, J 8.7 \mathrm{~Hz}, \mathrm{H}-2^{\prime}\right.$ and $\left.\mathrm{H}-6^{\prime},(18)\right], 6.86-6.83\left[0.4 \mathrm{H}, \mathrm{m}, \mathrm{H}-2^{\prime}\right.$ and H-6', (17)], 6.85 [2H, d, J $8.66 \mathrm{~Hz}, \mathrm{H}-2^{\prime}$ and H-6', (16)], 3.81-3.80 [3.9H, s, 4'-OMe, (16), (17) and (18)], 3.68 [3H, s, COOMe, (16)], $3.67[0.3 \mathrm{H}, \mathrm{s}, \mathrm{COOMe},(\mathbf{1 8})], 3.66[0.6 \mathrm{H}, \mathrm{s}, \mathrm{COOMe}(\mathbf{1 7})], 3.43[0.1 \mathrm{H}, \mathrm{t}, \mathrm{J} 7.73 \mathrm{~Hz}, \mathrm{H}-2,(18)], 2.98$ $[0.2 \mathrm{H}, \mathrm{dd}, J 6.94$ and $13.53 \mathrm{~Hz}, \mathrm{H}-3 \mathrm{a} / \mathrm{b},(17)], 2.75-2.69[0.2 \mathrm{H}, \mathrm{m}, \mathrm{H}-2,(17)], 2.65-2.63[0.2 \mathrm{H}, \mathrm{m}, \mathrm{H}-3 \mathrm{a} / \mathrm{b},(\mathbf{1 7})]$, $2.61[2 \mathrm{H}, \mathrm{t}, J 7.54 \mathrm{~Hz}, \mathrm{H}-4,(16)], 2.34[2 \mathrm{H}, \mathrm{t}, J 7.54 \mathrm{~Hz}, \mathrm{H}-2,(16)], 2.11-2.07[0.1 \mathrm{H}, \mathrm{m}, \mathrm{H}-3 \mathrm{a} / \mathrm{b},(18)], 1.94[2 \mathrm{H}, \mathrm{p}$, J $7.54 \mathrm{~Hz}, \mathrm{H}-3,(16)], 1.81-1.76[0.1 \mathrm{H}, \mathrm{m}, \mathrm{H}-3 \mathrm{a} / \mathrm{b},(18)], 1.16\left[0.6 \mathrm{H}, \mathrm{d}, J 6.92 \mathrm{~Hz}, 2-\mathrm{CH}_{3},(17)\right], 0.90[0.3 \mathrm{H}, \mathrm{t}, J 7.34$ $\mathrm{Hz}, \mathrm{H}-4,(\mathbf{1 8})] ; \delta_{\mathrm{C}}\left(151 \mathrm{MHz}, \mathrm{CDCl}_{3}\right) 176.7$ [C-1, (17)], 174.8 [C-1, (18)], 174.0 [C-1, (16)], 158.7 [C-4', (18)], 158.1 [C-4', (17)], 157.9 [C-4', (16)], 133.4 [C-1', (16)], 131.4 [C-1', (17)], 131.2 [C-1', (18)], 129.9 [C-2' and C-6', (17)], 129.4 [C-2' and C-6', (16)], 128.9 [C-2' and C-6', (18)], 113.9 [C-3' and C-5', (18)], 113.8 [C-3' and C-5', (16)], 113.7 [C-3' and C-5', (17)], 55.2 [4'-OMe, (16), (17) and (18)], 52.5 [C-2, (18)], 51.9 [COOMe, (18)], 51.6 [COOMe, (17)], 51.5 [COOMe (16)], 41.7 [C-2, (17)], 38.9 [C-3 (17)], 34.2 [C-4, (16)], 33.3 [C-2, (16)], 26.8 [C-3 (18)], 26.7 [C-3, (16)], 16.7 [2- $\left.\mathrm{CH}_{3}(\mathbf{1 7})\right], 12.2$ [C-4, (18)]; MS (EI) m/z $208\left[\mathrm{M}^{+}, 39 \%,(16)\right], 208\left[\mathrm{M}^{+}, 13 \%,(17)\right]$, $208\left[\mathrm{M}^{+}, 21 \%,(18)\right]$.

Methyl 4-(2'-methoxyphenyl)butanoate ${ }^{27}$ (19), methyl 3-(2'-methoxyphenyl)-2-methylpropanoate ${ }^{24}$ (20) and methyl 2-(2'-methoxyphenyl)butanoate ${ }^{17}$ (21). $\mathrm{R}_{\mathrm{f}} 0.583$ (petroleum ether:ether 7:3); $\delta_{\mathrm{H}}\left(600 \mathrm{MHz} \mathrm{CDCl}_{3}\right.$; $\left.\mathrm{Me}_{4} \mathrm{Si}\right)$ 7.25-7.23 [0.04H, m, H-4', (21)], 7.20-7.15 [1.2H, m, H-4', (19) and (20)], 7.11-7.07 [1.25H, m, H-6', (19), (20) and (21)], 6.94-6.91 [0.04H, m, H-5', (21)], 6.88-6.84 [1.2H, m, H-5', (19) and (20)], 6.83-6.81 [1.25H, m, H3', (19), (20) and (21)], 3.80 [0.12H, s, 4'-OMe, (21)], 3.79 [0.6H, s, 4'-OMe, (20)], 3.78 [3H, s, 4'-OMe, (19)], 3.76-3.75 [0.04H, m, H-2, (21)], 3.64 [3H, s, COOMe, (19)], 3.63 [0.12H, s, COOMe, (21)], 3.61 [0.6H, s, COOMe (20)], $2.98[0.2 \mathrm{H}, \mathrm{dd}, J 7.10$ and $13.22 \mathrm{~Hz}, \mathrm{H}-3 \mathrm{a} / \mathrm{b},(20)], 2.86-2.80[0.2 \mathrm{H}, \mathrm{m}, \mathrm{H}-2,(20)], 2.70[0.2 \mathrm{H}, \mathrm{dd}, J 7.59$ and $13.22 \mathrm{~Hz}, \mathrm{H}-3 \mathrm{a} / \mathrm{b},(20)], 2.65[2 \mathrm{H}, \mathrm{t}, J 7.56 \mathrm{~Hz}, \mathrm{H}-4,(19)], 2.32[2 \mathrm{H}, \mathrm{t}, J 7.56 \mathrm{~Hz}, \mathrm{H}-2,(19)], 2.08-2.03[0.04 \mathrm{H}$, m, H-3a/b, (21)], $1.92[2 \mathrm{H}, \mathrm{p}, J 7.56 \mathrm{~Hz}, \mathrm{H}-3,(19)], 1.78-1.73[0.04 \mathrm{H}, \mathrm{m}, \mathrm{H}-3 \mathrm{a} / \mathrm{b},(21)], 1.13[0.6 \mathrm{H}, \mathrm{d}, J 7.0 \mathrm{~Hz}, 2-$ $\left.\mathrm{CH}_{3},(20)\right], 0.89[0.12 \mathrm{H}, \mathrm{t}, J 7.39 \mathrm{~Hz}, \mathrm{H}-4,(21)] ; \delta_{\mathrm{C}}\left(151 \mathrm{MHz}, \mathrm{CDCl}_{3}\right) 177.1$ [C-1, (20)], 175.0 [C-1, (21)], 174.2 [C1, (19)], 157.6 [C-2', (20)], 157.5 [C-2', (19)], 156.9 [C-2', (21)], 130.8 [C-6', (20)], 131.0 [C-6', (19)], 129.7 [C-1', (19)], 129.4, 128.4, 128.1, 127.72, 127.69 [C-4', (20)], 127.3 [C-4' (19)], 120.7 [C-5', (21)], 120.4 [C-5', (19)], 120.3 [C-5', (20)], 110.7 [C-3', (21)], 110.2 [C-3', (19) and (20)], 55.7 [2'-OMe, (21)], 55.2 [2'-OMe, (19) and (20)], 51.8 [COOMe, (21)], 51.47 [COOMe, (20)], 51.45 [COOMe (19)], 45.7 [C-2, (21)], 39.5 [C-2, (20)], 34.6 [C$3(20)], 33.6$ [C-2, (19)], 29.5 [C-4, (19)], 25.7 [C-3 (21)], 25.0 [C-3, (19)], 17.0 [2-CH $(20)], 12.2$ [C-4, (21)]; MS (EI) $m / z 208\left[\mathrm{M}^{+}, 40 \%,(19)\right], 208\left[\mathrm{M}^{+}, 22 \%,(20)\right], 208\left[\mathrm{M}^{+}, 34 \%,(21)\right]$. 


\section{Acknowledgements}

Financial support by Sasol Ltd and the National Research Foundation of South Africa is greatly appreciated.

\section{Supplementary Material}

Quantities of reagents for the methoxycarbonylation of substrates under microwave conditions and ${ }^{1} \mathrm{H}$ and ${ }^{13} \mathrm{C}$ NMR spectra of mixtures of linear, branched products.

\section{References}

1. Knifton, J.F. J. Org. Chem. 1976, 41, 2885-2890.

https://doi.org/10.1021/jo00879a020

2. Sugi, Y.; Bando, K. Chem. Lett. 1976, 727-730.

https://doi.org/10.1246/cl.1976.727

3. Cometti, G.; Chiusoli, G.P.; J. Organomet. Chem. 1982, 236, C31-C32.

https://doi.org/10.1016/S0022-328x(00)86766-2

4. Huh, K.-T.; Alper, H. Bull. Korean Chem. Soc. 1994, 15, 304-306.

5. Yun, H.S.; Lee, K.H.; Lee, J.S. J. Mol. Catal. A: Chem. 1995, 95, 1 https://doi.org/10.1016/1381-1169(94)00150-2

6. Lee, C.W.; Alper, H. J. Org. Chem. 1995, 60, 250-252.

https://doi.org/10.1021/jo00106a042

7. Oi, S.; Nomura, M.; Aiko, T.; Inoue, Y. J. Mol. Catal. Chem. 1997, 115, 289-295. https://doi.org/10.1016/S1381-1169(96)00282-8

8. Nozaki, K.; Kantam, M.L.; Horiuchi, T.; Takaya, H. J. Mol. Catal. Chem. 1997, 118, 247-253. https://doi.org/10.1016/S1381-1169(96)00385-8

9. Mukhopadhyay, K.; Sarkar, B.R.; Chaudhari, R.V. J. Am. Chem. Soc. 2002, 124, 9692-9693. https://doi.org/10.1021/ja025991q

10. Rodrigues, C.J.; Foster, D.F.; Eastham, G.R.; Cole-Hamilton, D.J. Chem. Commun. 2004, 1720-1721. https://doi.org/10.1039/B404783D

11. Kawashima, Y.; Okano, K.; Nozaki, K.; Hiyama, T. Bull. Chem. Soc. Jpn. 2004, 77, 347-355. https://doi.org/10.1246/bcsj.77.347

12. Vieira, T.O.; Green, M.J.; Alper, H. Org. Lett. 2006, 8, 6143-6145. https://doi.org/10.1021/ol062646n

13. Guiu, E.; Caporali, M.; Muñoz, B.; Müller, C.; Lutz, M.; Spek, A.L.; Claver, C. Van Leeuwen, P.W.N.M. Organometallics, 2006, 25, 3102-3104. https://doi.org/10.1021/om060121t

14. Muñoz, B.K.; Godard, C.; Marinetti, A.; Ruiz, A.; Benet-Buchholz, J.; Claver, C. Dalton Trans. 2007, 55245530.

https://doi.org/10.1039/B705230H 
15. Zolezzi, S.; Moya, S.A.; Valdebenito, G.; Abarca, G.; Parada, J.; Aguirre, P. Appl. Organomet. Chem. 2014, 28, 364-371.

https://doi.org/10.1002/aoc.3137

16. Schneider, C.; Jackstell, R.; Maes, B.U.W.; Beller, M. Eur. J. Org. Chem. 2020, 2020(8), 932-936. https://doi.org/10.1002/ejoc.201901592

17. Du Plessis, M.; Marais, C.; Bezuidenhoudt, B.C.B. Synthesis, 2016, 48, 557-565.

https://doi.org/10.1055/s-0035-1560912

18. Hayes, B. L. Microwave Synthesis: Chemistry at the Speed of Light, CEM Publishing, 2002.

19. Williams, D.B.G.; Shaw, M.L.; Green, M.J.; Holzapfel, C.W. Angew. Chem. Int. Ed., 2008, 47, 560-563. https://doi.org/10.1002/anie.200702889

20. Williams D.B.G.; Bredenkamp, T.; Chem. Cat. Chem., 2012, 4, 206-208. https://doi.org/10.1002/cctc.201100238

21. Pfeffer, P. E.; Foglia, T. A.; Barr, P. A.; Obenauf, R. H. J. Org. Chem. 1978, 43, 3429-3431. https://doi.org/10.1021/jo0041a051

22. Hayashi, M.; Kawabata, H.; Yoshimoto, K.; Tanaka, T. Phosphorus Sulfur Silicon Relat. Elem. 2007, 182, 433-445. https://doi.org/10.1080/10426500600977296

23. Marsh, B. J.; Carbery, D. R. J. Org. Chem. 2009, 74, 3186-3188. https://doi.org/10.1021/jo900237y

24. Liu, Y.; Mao, D.; Xu, D.; Xu, Z.; Zhang, Y. Synth. Commun. 2007, 37, 4389-4397. https://doi.org/10.1080/00397910701577570

25. Shono, T.; Kashimura, S.; Nogusa, H. J. Org. Chem. 1984, 49, 2043-2045. https://doi.org/10.1021/jo00185a052

26. Malézieux, B.; Gruselle, M.; Troitskaya, L.; Sokolov, V. Tetrahedron Asymmetry 1998, 9, 259-269. https://doi.org/10.1016/S0957-4166(97)00636-8

27. Haine, M. E.; Dyson, J. Nature 1950, 166 (4216), 315-316. https://doi.org/10.1038/166315a0 